\title{
Effect of molding helmets on intracranial pressure and head shape in nonsurgically treated sagittal craniosynostosis patients
}

\author{
Neena I. Marupudi, MD, MS, Sandeep Sood, MD, Arlene Rozzelle, MD, and Steven D. Ham, DO \\ Children's Hospital of Michigan, Wayne State University School of Medicine, Detroit, Michigan
}

\begin{abstract}
OBJECTIVE Cranial vault expansion is performed in pediatric patients with craniosynostosis to improve head shape. Another argument for performing total cranial vault reconstruction is the potential reduction in the harmful effects of elevated intracranial pressure (ICP) that are associated with craniosynostosis. Alternatively, molding helmets have been shown to improve the cranial index $(\mathrm{Cl})$ in patients with sagittal synostosis without surgery. However, it is unknown if the use of molding helmets without surgery contributes to adverse changes in ICP. The effect of molding helmets on ICP and $\mathrm{Cl}$ in patients with sagittal synostosis was investigated.
\end{abstract}

METHODS A prospective cohort study of 24 pediatric patients with sagittal synostosis who planned to undergo total cranial reconstruction was performed from 2011 to 2014 at the Children's Hospital of Michigan. A preoperative molding helmet was used in 13 patients, and no molding helmet was used in 11 patients. End-tidal carbon dioxide, patient positioning, level of sedation, type of anesthetic, and the monitoring site at the time of intraoperative recording were regulated and standardized to establish the accuracy of the ICP readings. $\mathrm{Cl}$ and head circumference were monitored for each patient.

RESULTS The mean duration of the preoperative use of the molding helmet was 17 weeks (range 7-37 weeks). Under controlled settings, the average intraoperative ICP was $7.2 \mathrm{~mm} \mathrm{Hg}$ (range 2-18 mm Hg) for patients treated with a preoperative molding helmet and $9.5 \mathrm{~mm} \mathrm{Hg}$ (range $2-22 \mathrm{~mm} \mathrm{Hg}$ ) for patients with no preoperative molding helmet. ICP was not significantly different between the 2 groups, suggesting that the use of a molding helmet in this population is safe. The average $\mathrm{Cl}$ at the time of helmet placement was 0.70 (range $0.67-0.73$ ), and this improved to an average of 0.74 (range 0.69-0.79) after using the molding helmet for a mean of 17 weeks.

CONCLUSIONS ICPS were not significantly different with the use of a preoperative molding helmet, refuting the prevailing thought that molding helmets would be detrimental in children who have craniosynostosis. The use of molding helmet in this population of patients improves head shape and does not adversely affect ICP.

http://thejns.org/doi/abs/10.3171/2016.1.PEDS15569

KEY WORDS molding helmet; craniosynostosis; intracranial pressure; scaphocephaly; sagittal synostosis; craniofacial

$\mathrm{T}$ HE proposed goals of cranial vault expansion surgery in pediatric patients with craniosynostosis include improving head shape and potentially reducing the harmful effects associated with elevated intracranial pressure (ICP). The surgical options for sagittal synostosis include total cranial reconstruction (TCR) and endoscopic strip craniectomy. In many cases, molding helmets are used in the postoperative period to further improve cosmesis. Alternatively, molding helmets can be used to improve the cranial index (CI) in patients with sagittal synostosis without surgery. We previously published a cases series demonstrating improved head shape with the use of a molding helmet but without surgical intervention. ${ }^{6}$ However, the use of molding helmets without surgery remains controversial due to concerns regarding the putative adverse effects on ICP. This study was done to answer criticisms regarding the potential increase in ICP with the use of molding helmets in patients with untreated sagittal craniosynostosis.

We investigated the effect of molding helmets on ICP in patients with sagittal synostosis. Our results suggest that in patients with scaphocephaly who have not had craniosynostosis surgery, the use of a molding helmet improves head shape and does not adversely affect ICP.

ABBREVIATIONS $\mathrm{Cl}=$ cranial index; $\mathrm{ICP}=$ intracranial pressure; $\mathrm{MAC}=$ minimum alveolar concentration; $\mathrm{REM}=$ rapid eye movement; $\mathrm{TCR}=$ total cranial reconstruction. SUBMITTED September 21, 2015. ACCEPTED January 13, 2016.

INCLUDE WHEN CITING Published online April 8, 2016; DOI: 10.3171/2016.1.PEDS15569. 


\section{Methods}

This prospective cohort study was performed in patients who presented with isolated sagittal craniosynostosis to the Children's Hospital of Michigan between 2011 and 2014. Under an institutional review board-approved protocol, a molding helmet was used on patients younger than 3 months of age if their parents consented and elected to undergo total calvarial reconstruction in preference over endoscopic craniectomy and in patients 3 months or older who were predetermined to undergo TCR. The parents of the patients were informed of the following at the time of recruitment. 1) The surgical release of sutures/reconstruction is the standard of care for the management of sagittal craniosynostosis. 2) The role of early surgery (endoscopic strip craniectomy) versus late surgery (calvarial reconstruction) in terms of risks (e.g., surgical anesthesia) and benefits (e.g., better head shape, reoperations, late restriction of head growth, increased ICP) is not well established in literature. 3) We have observed improvement in head shape using a molding helmet without surgery. 4) There is no study in literature that supports the notion that molding helmets are detrimental to the growth of the head unless they are ill fitting or that molding helmets have any negative effect on the development of the brain in patients with sagittal craniosynostosis. Based on this, the only risk that we anticipated was the development of pressure sores. For this reason, the patients are monitored in the clinic every 3 to 4 weeks, as is routine for all patients who have a molding helmet. 5) The benefit of using a molding helmet could be a significant improvement in head shape.

All patients included in this study had a diagnosis of sagittal synostosis based on their CI measurements (maximum biparietal width of the head divided by the maximum length) and underwent a CT scan of the head with reconstruction to confirm the diagnosis. Patients were evaluated by both a pediatric neurosurgeon and a pediatric plastic surgeon and monitored for head circumference, CI, and developmental milestones every 3 to 4 weeks. Helmet adjustments were done if needed at that time. If the patient outgrew the helmet prior to the planned surgery, the helmet was discontinued and a new helmet was instituted only after surgery.

All patients who presented to us with sagittal craniosynostosis and were younger than 6 months were approached for the study. Of these 25 patients, 14 were 3 months or younger, and the parents of 8 patients consented to participation in the study. These patients underwent helmet therapy prior to TCR. Of 11 patients between 3 and 6 months of age at presentation, the parents of 5 patients consented to their participation in this study and their children had a helmet placed prior to TCR, whereas 6 patients whose parents did not consent to participation in the study underwent TCR without a helmet. These 6 patients, along with 5 patients who had presented after 6 months, were used as controls for the ICP measurements obtained at surgery. Overall, we had 24 pediatric patients with sagittal synostosis who planned to undergo TCR for craniosynostosis. A preoperative molding helmet was used in 13 patients (age range at helmet placement 2-5.1 months), and no preoperative molding helmet was used in 11 patients (age range at surgery 6.1-36.1 months). All patients underwent TCR, but just prior to craniotomy ICP recording was performed under a controlled setting. All patients in the study were subsequently placed in a molding helmet postoperatively, regardless of whether they were treated with a helmet preoperatively.

The statistical analysis was performed using the Student t-test. The outcome of interest was if the sagittal synostosis patients who were treated with a molding helmet had higher ICP than those patients treated without a molding helmet preoperatively, which was based on the premise that if the helmet restricts the growth of the head then it would cause an increase in ICP that would be present even when the helmet was removed at the time of surgery.

\section{Procedure for ICP Measurement}

ICP measurements are routinely taken in all patients at the time of TCR at our institution. All calvarial reshaping procedures were performed in conjunction with our reconstructive surgery specialist. After a bicoronal skin incision was completed by the reconstructive surgeon, the pediatric neurosurgeon placed bur holes for bifrontal and biparietal craniotomies. An ICP reading was intraoperatively performed just prior to performing the craniotomy. End-tidal carbon dioxide, patient positioning, level of sedation, type of anesthetic, and the monitoring site at the time of the intraoperative recording were regulated and standardized to establish the accuracy of the ICP readings.

End-tidal carbon dioxide was maintained at $35 \mathrm{~mm}$ $\mathrm{Hg}$ during the recording of the ICP. All patients were positioned in the prone position for the calvarial reshaping procedure. Isoflurane was consistently used as the inhaled anesthetic in all patient procedures. A moderate level of sedation was maintained at a level of 1.5 minimum alveolar concentrations (MACs) of isoflurane during the pressure recordings. The pressure sensor (Codman ICP Express) was placed through the parietal bur hole into the intraparenchymal or subdural space (Table 1). Of note, the parietal bur hole was made as part of the planned craniectomy for calvarial reshaping. The ICP monitor was brought out through a separate stab incision in the skin and secured into place with sutures for monitoring throughout the surgical procedure and postoperatively. The reconstructive surgeon subsequently completed the calvarial reshaping and replaced the molded bone.

\section{Results}

From 2011 to 2014, 24 patients (17 male and 7 female patients) with sagittal synostosis underwent TCR. Thir-

TABLE 1. Standardized variables for accurate and consistent ICP recording under controlled settings

\begin{tabular}{ll}
\hline \multicolumn{1}{c}{ Variable } & \multicolumn{1}{c}{ Setting Description } \\
\hline End-tidal carbon dioxide & $35 \mathrm{~mm} \mathrm{Hg}$ \\
\hline Patient positioning & Prone \\
\hline Anesthesia & Isoflurane \\
\hline Level of sedation & $1.5 \mathrm{MAC}$ \\
\hline Type of ICP sensor & Strain gauge (Codman ICP Express) \\
\hline Monitoring site & Parietal bur hole \\
\hline
\end{tabular}




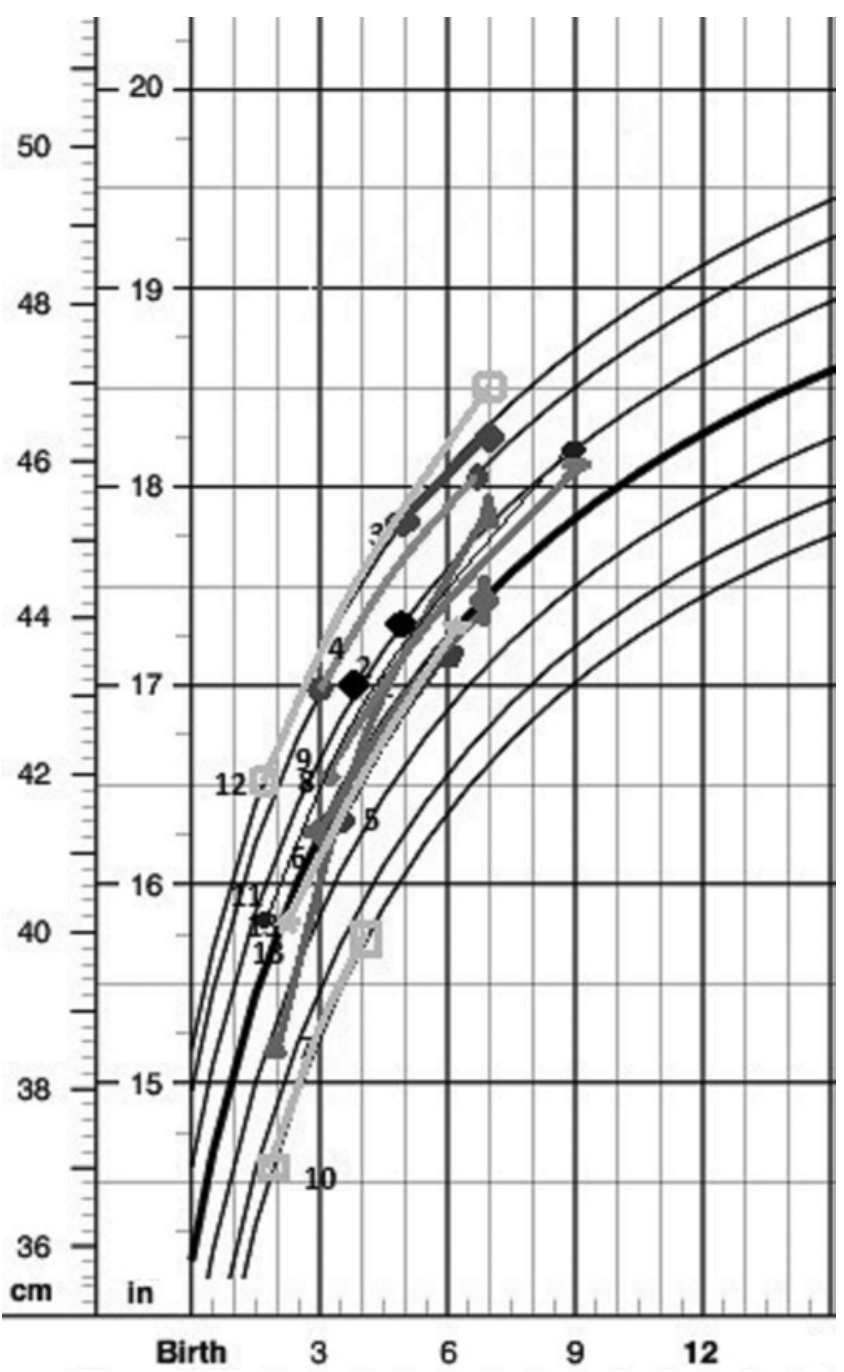

FIG. 1. Head circumference curves of the patients who received a molding helmet showing that the patients maintained the centiles.

teen patients (54\%) were placed in a molding helmet for up to 6 months prior to surgery. Eleven patients (46\%) were not treated with a molding helmet prior to synostosis surgery. All patients were placed in a molding helmet for approximately 2 weeks after the calvarial reshaping procedure. The mean duration of preoperative use of a molding helmet was 17 weeks (range 7-37 weeks). No patient had any adverse effects such as pressure sores from the use of the helmet. Overall, ICP was lower than $10 \mathrm{~mm} \mathrm{Hg}$ in 17 patients. Three patients had ICP higher than $15 \mathrm{~mm} \mathrm{Hg}$.

Of the 13 patients treated with a preoperative molding helmet, no patient had worsening CI measurements or a decrease in the head circumference growth rate from the time of placement in the molding helmet to the time of surgery (Fig. 1). One patient was noncompliant with the use of the molding helmet and had minimal improvement in CI; of the other 2 patients who had limited improvement in CI, 1 patient probably had an underlying undiagnosed syndrome (Fig. 2) and the other patient was more than 3 months old at the time of receiving the helmet. Nine patients had substantial improvements in head shape and CI

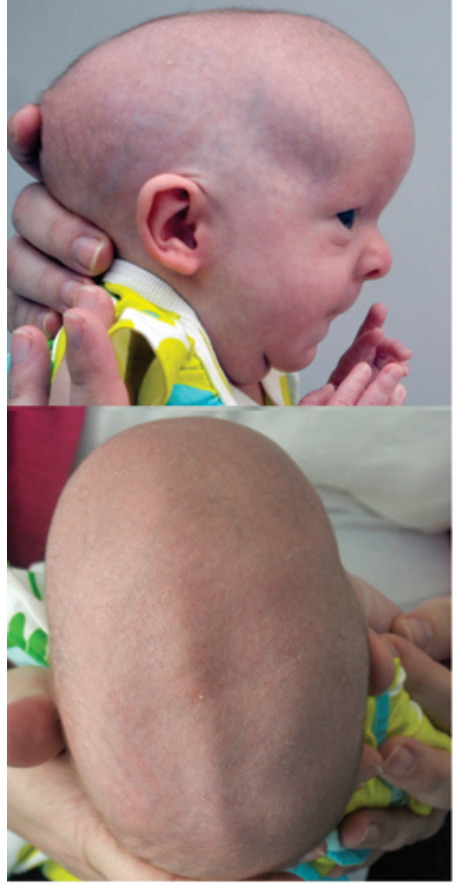

Pre Helmet

( $1.5 \mathrm{mth} ; \mathrm{Cl} 0.67$ )
Post Helmet

(4.5 mth; Cl 0.69)

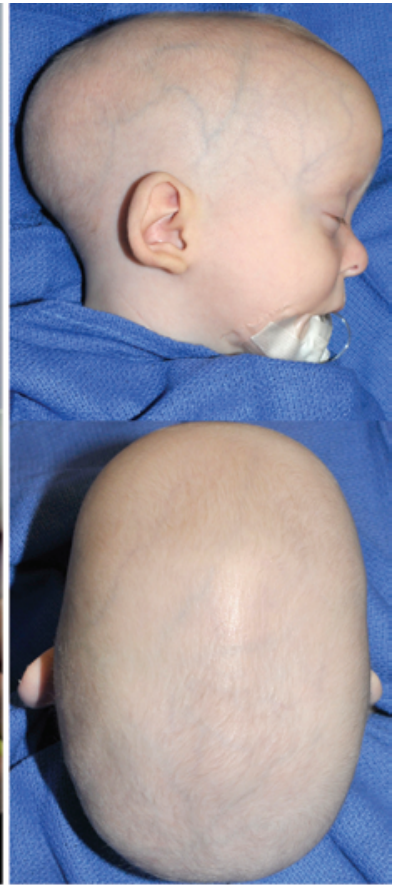

FIG. 2. Pre- and posthelmet photographs obtained in a patient who exhibited only minimal improvement in shape and $\mathrm{Cl}$. $\mathrm{mth}=$ months. Figure is available in color online only.

(Fig. 3). For this group (Table 2), the average CI at the time of placement in the helmet was 0.70 (range 0.67-0.73). The average CI at time of surgery (i.e., after treatment with a molding helmet) was 0.74 (range 0.69-0.79). This difference was significant at $p<0.001$. As expected, all patients had improved CI scores after calvarial reshaping. The average postoperative $\mathrm{CI}$ was 0.83 (range $0.78-0.90$ ).

Of the 11 patients who were not placed in a preoperative molding helmet, the average $\mathrm{CI}$ at the time of surgery was 0.70 (range $0.64-0.77$ ). The average postoperative CI was 0.81 (range $0.77-0.87$ ) (Table 2).

Under controlled, optimized, and consistent conditions, the average intraoperative ICP was $7.2 \mathrm{~mm} \mathrm{Hg}$ (range 2-18 $\mathrm{mm} \mathrm{Hg}$ ) in patients treated with a preoperative molding helmet and $9.5 \mathrm{~mm} \mathrm{Hg}$ (range $2-22 \mathrm{~mm} \mathrm{Hg}$ ) in patients with no preoperative molding helmet (Tables 3 and 4). ICP was not significantly different between the 2 groups $(\mathrm{p}=$ 0.37). Postoperatively, ICP remained in the normal range in all patients.

There was no correlation between CI at presentation and ICP measurements made at surgery for patients who received a helmet $\left(R^{2}=0.01\right)$ or those who did not $\left(R^{2}\right.$ $=0.00)$. Likewise, there was no correlation between age at presentation and ICP measurements in either of the 2 groups $\left(R^{2}=0.13\right.$ and $R^{2}=0.00$, respectively)

\section{Discussion}

The current treatment for sagittal synostosis does not include the trial use of a molding helmet prior to craniosyn- 


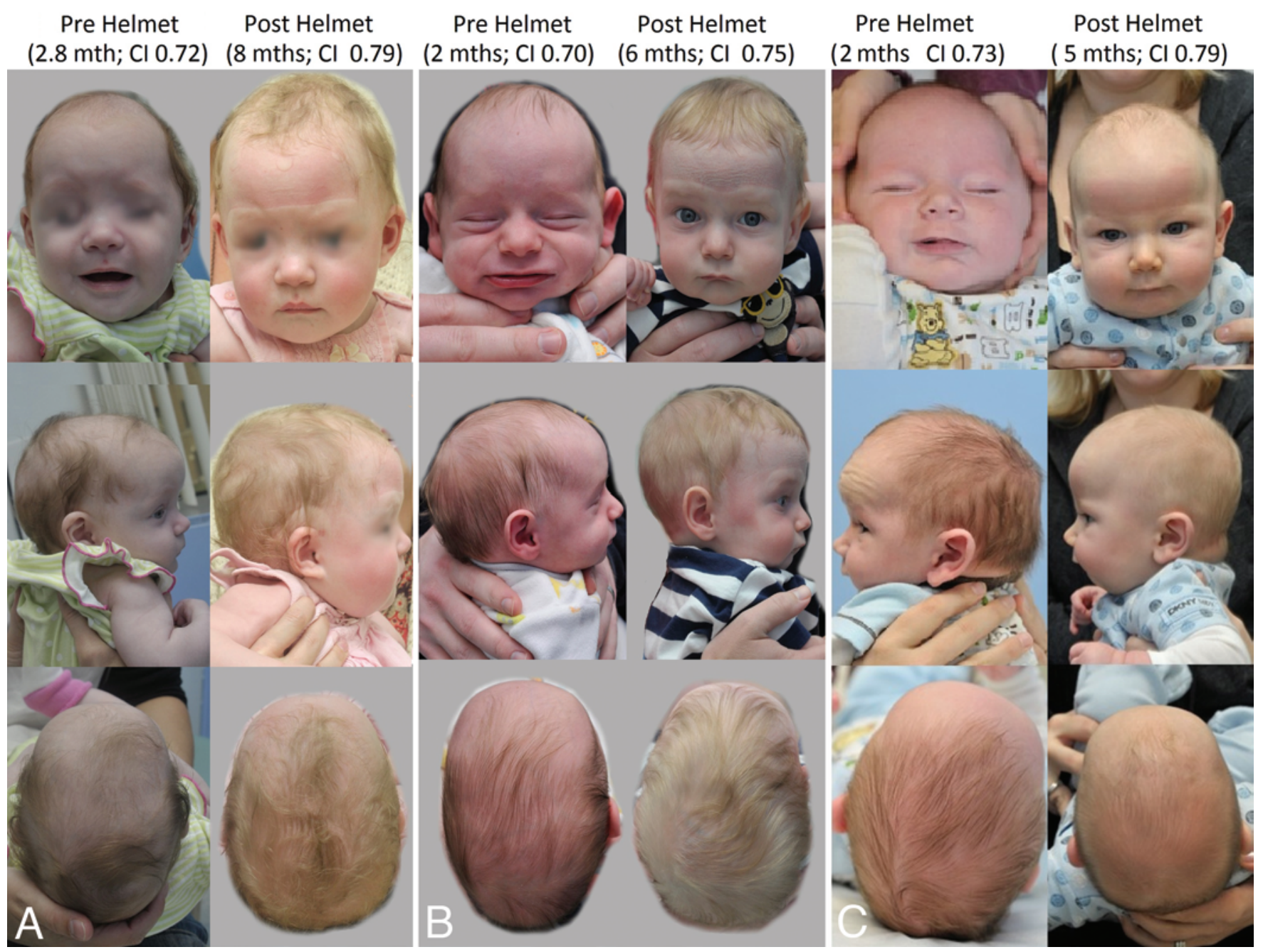

FIG. 3. Pre- and post-helmet photographs patients showing improvement in shape and $\mathrm{Cl}$, decreased forehead bossing (Patient $\mathrm{A})$, an increase in the posterior vertex height, and improvement in dolichocephaly (Patients A-C). Figure is available in color online only.

ostosis surgery. This may be due to concerns that helmet use without surgical correction could lead to constricted cranial growth and elevated ICP. This study confirms that a molding helmet used prior to calvarial reconstruction for synostosis does not result in adverse effects, namely elevated ICP, and at the same time improves head shape. Furthermore, our ICP measurements in scaphocephaly patients did not reveal any age-based correlation or correlations to the CI measurements. More severe CI scores in scaphocephaly patients did not result in elevated or higher ICP values. The use of a molding helmet on the patient with a lower CI score (i.e., more severe scaphocephaly) did not correlate with elevated ICP at the time of intraoperative recording. This suggests that the severity of sagittal craniosynostosis may not be considered a deterrent to using molding helmets to achieve head shape correction. While many patients had significant improvement in the head shape, the decision to operate was mainly driven by parental concern and fear of restricting head growth over the long term because of the closed sutures.

Even though end-tidal carbon dioxide was maintained at $35 \mathrm{~mm} \mathrm{Hg}$, an argument may be made that ICP mea- sured under anesthesia may be falsely high due to the effects of anesthetics and may not reflect the true pressure. Nonetheless, since the mean pressures were not high in either group, this argument may not be valid in our patient cohort. It may also be argued that we measured ICP only for a brief period, and a longer duration of monitoring such as for 24 hours may be a more accurate guide. This type of long-duration monitoring may show Type A and Type B waves that are reportedly associated with pathologically abnormal ICP. While no normative data on long-term monitoring exist in children and adults, the idea that these waveforms reflect an abnormality has been extrapolated from recordings obtained in patients who have an intracranial pathology. Interestingly, Wayenberg et al. ${ }^{11}$ have shown that plateau waves and B waves are normal in infants and occur frequently during rapid eye movement (REM) sleep. In a larger study by Anegawa et al. ${ }^{1}$ on 32 infants, the same conclusion was drawn. After comparing these data to data for 42 hydrocephalic patients, these authors concluded that Type I waves (i.e., Lundberg Type A) lack the consistency needed to effectively discriminate between normal and abnormal ICP during sleep in infants. ${ }^{2}$ 
TABLE 2. $\mathrm{Cl}$ in sagittal synostosis patients treated without and with a preoperative molding helmet for a mean of $17.4 \pm 7.4$ weeks prior to surgery*

\begin{tabular}{lcc}
\hline \multicolumn{1}{c}{ Variable } & w/ Preop Helmet & w/o Preop Helmet \\
\hline No. of patients & 13 & 11 \\
\hline Age (mos) & & \\
\hline At helmet placement & $2.9 \pm 0.8$ & $11.1 \pm 8.4$ \\
\hline At op & \\
Cl & \\
\hline Prehelmet therapy & $0.70 \pm 0.02$ & \\
\hline Posthelmet therapy & $0.74 \pm 0.03 \dagger$ & $0.70 \pm 0.03$ \\
\hline Preop & & $0.81 \pm 0.03 \dagger$ \\
\hline Postop & &
\end{tabular}

* Values are shown as the mean \pm standard deviation unless otherwise indicated.

$\dagger$ Significant at $p<0.001$.

They further stated that the appearance of Type II waves (similar to Lundberg Type B waves, which occur normally in infants and only in REM sleep) in non-REM sleep could be an initial sign of a low-compliance state and does not represent an abnormality in ICP. We, therefore, feel that a longer duration of monitoring in infants may not be needed to access ICP and likely would not have changed our results. Prior to this study, there was only 1 other small series where ICP measurements were performed on a variety of patients with craniosynostosis under controlled settings, including regulated end-tidal carbon dioxide, type of sedation and level, positioning, and monitoring site..$^{12}$ Our paper presents the first series to evaluate ICP in sagittal synostosis under controlled conditions with and without the use of a preoperative molding helmet.

Preoperative and postoperative ICP recordings that compare nonsyndromic and syndromic craniosynostosis suggest that syndromic patients most commonly have an associated increase in ICP. ${ }^{8}$ On the other hand, the incidence of elevated ICP in single-suture synostosis remains controversial in the literature, with reported numbers varying from $10 \%$ to $44 \%$. In a heterogeneous group of patients with craniosynostosis, Eide et al. reported abnormally increased ICP (i.e., $>15 \mathrm{~mm} \mathrm{Hg}$ ) in $26.4 \%$ of patients and a weak correlation between age and mean ICP based on the ICP recordings obtained during sleep. ${ }^{4}$ Thompson et al. reported an increase in ICP in only $17 \%$ of cases but an inverse correlation between age and ICP, particularly in patients with scaphocephaly and trigonocephaly. ${ }^{9}$ A recent publication by Wall et al. suggests that $44 \%$ of patients with sagittal synostosis, who did not undergo surgery in infancy, had increased ICP when measured at an older age..$^{10}$ They used an ICP threshold of 15 $\mathrm{mm} \mathrm{Hg}$ or the presence of more than $3 \mathrm{~B}$ waves in a 24hour period.

Much of this controversy centers on what is considered normal ICP in infants and children. Noninvasive fontanelle pressure measurements in normal infants are the only available data we have for comparison., ${ }^{1,2,11}$ According to these data, ${ }^{11}$ during quiet sleep in infants older than 4 months, the highest pressures were $9.7 \pm 1.9 \mathrm{~mm}$
TABLE 3. Controlled-setting ICP measurements in patients with sagittal synostosis

\begin{tabular}{|c|c|c|}
\hline Patient No. & Preop Molding Helmet & Intraop ICP (mm Hg) \\
\hline 1 & Yes & 18 \\
\hline 2 & Yes & 15 \\
\hline 3 & Yes & 11 \\
\hline 4 & Yes & 8 \\
\hline 5 & Yes & 8 \\
\hline 6 & Yes & 7 \\
\hline 7 & Yes & 6 \\
\hline 8 & Yes & 5 \\
\hline 9 & Yes & 4 \\
\hline 10 & Yes & 4 \\
\hline 11 & Yes & 4 \\
\hline 12 & Yes & 2 \\
\hline 13 & Yes & 2 \\
\hline 14 & No & 22 \\
\hline 15 & No & 20 \\
\hline 16 & No & 15 \\
\hline 17 & No & 13 \\
\hline 18 & No & 12 \\
\hline 19 & No & 9 \\
\hline 20 & No & 4 \\
\hline 21 & No & 3 \\
\hline 22 & No & 3 \\
\hline 23 & No & 2 \\
\hline 24 & No & 2 \\
\hline
\end{tabular}

Hg. In transient sleep and REM sleep, the highest pressures were $11.3 \pm 2.3 \mathrm{~mm} \mathrm{Hg}$. In an infant undergoing pressure recording during sleep, interpreting ICP without knowledge of the sleep stage may be difficult. Nonetheless, if the sleep stage is unknown, ICP greater than 11.3 +3 standard deviations (i.e., $18 \mathrm{~mm} \mathrm{Hg}$ ) at any time may be considered abnormal. It may, therefore, be reasonable to consider baseline pressures greater than $18 \mathrm{~mm} \mathrm{Hg}$ in infants older than 4 months as abnormal. In addition, the usefulness of ICP criteria involving B or A waves in infants may be questionable since studies show that these waves occur normally during REM sleep in infants. It is also controversial if the pressures in this range need to be treated if the child is asymptomatic. Although prior publications suggest that the need for surgery should be considered in these patients based on the hypothesis that pressures in this range could potentially affect cognitive development at a later date, there is no convincing evidence in the literature to support this view.

TABLE 4. Effect of a preoperative molding helmet on ICP

\begin{tabular}{ccc}
\hline $\begin{array}{c}\text { Preop Molding } \\
\text { Helmet }\end{array}$ & $\begin{array}{c}\text { Average ICP, } \\
\mathrm{mm} \mathrm{Hg}\end{array}$ & $\begin{array}{c}\text { Median ICP } \\
\text { (range), mm Hg }\end{array}$ \\
\hline Yes $(n=13)$ & 7.2 & $5(2-18)$ \\
\hline No $(n=11)$ & 9.5 & $9(2-22)$ \\
\hline
\end{tabular}


Clear guidelines, based on ICP criteria, on when and what surgical intervention should take place have not been established for sagittal craniosynostosis. ${ }^{7}$ The studies on single-suture craniosynostosis that have included measurements of both ICP and neurodevelopmental status have failed to find any reliable association between the two. ${ }^{3,5}$ Without a solid foundation that establishes a relationship between symptoms, neurodevelopment, and ICP and an understanding of the range of numbers, we feel that decision-making guidelines for the treatment of sagittal craniosynostosis should not be based on ICP measurements alone.

For patients with sagittal craniosynostosis, if the head shape remains the cornerstone for the utility of surgery, and considering our data, which suggest no adverse effects on ICP from the use of helmets, the role of molding helmets should be further explored in this patient population. We have noted qualitative improvements in our scaphocephalic patients treated with molding helmets: increased $\mathrm{CI}$, widening and flattening of the occipital protrusion, decreased forehead bossing, and increase in the posterior vertex height. ${ }^{6}$ Molding helmets may not produce the best outcome in every case, but if the use of molding helmets does not have adverse effects then the use of preoperative helmets may help avoid surgery in select patients or allow for less radical surgical techniques for correction in other patients.

This is a prospective, nonrandomized study comprising a small group of patients; nevertheless, this study brings to attention the fact that molding helmets are not associated with an increase in ICP even when used in patients with untreated sagittal craniosynostosis. It substantiates our previous observation that even a short period of using molding helmets is effective in improving head shape when used prior to 3 months of age in patients with sagittal craniosynostosis who have not been operated on. It remains to be seen if the continued use of molding helmets beyond the period in this study would be additionally beneficial.

\section{Conclusions}

The use of molding helmets may effectively improve head shape in patients with sagittal craniosynostosis. ICP was not significantly different with the use of a preoperative molding helmet, refuting the prevailing thought that molding helmets would be detrimental to children who have craniosynostosis.

\section{References}

1. Anegawa S, Hayashi T, Torigoe R: Clinical significance of ICP measurement in infants. Part I: Normal intracranial pressure, in Matsumoto S, Tamaki N (eds): Hydrocephalus: Pathogenesis and Treatment. Tokyo: Springer, 1991, pp 248-257

2. Anegawa S, Hayashi T, Torigoe R: Clinical significance of ICP measurement in infants. Part II: Intracranial pressure in infants with hydrocephalus, in Matsumoto S, Tamaki N (eds):
Hydrocephalus: Pathogenesis and Treatment. Tokyo: Springer, 1991, pp 257-269

3. Arnaud E, Renier D, Marchac D: Prognosis for mental function in scaphocephaly. J Neurosurg 83:476-479, 1995

4. Eide PK, Helseth E, Due-Tønnessen B, Lundar T: Assessment of continuous intracranial pressure recordings in childhood craniosynostosis. Pediatr Neurosurg 37:310-320, 2002

5. Gewalli F, Guimarães-Ferreira JP, Sahlin P, Emanuelsson I, Horneman G, Stephensen H, et al: Mental development after modified pi procedure: dynamic cranioplasty for sagittal synostosis. Ann Plast Surg 46:415-420, 2001

6. Sood S, Rozzelle A, Shaqiri B, Sood N, Ham SD: Effect of molding helmet on head shape in nonsurgically treated sagittal craniosynostosis. J Neurosurg Pediatr 7:627-632, 2011

7. Tamburrini G, Caldarelli M, Massimi L, Santini P, Di Rocco $\mathrm{C}$ : Intracranial pressure monitoring in children with single suture and complex craniosynostosis: a review. Childs Nerv Syst 21:913-921, 2005

8. Thompson DN, Harkness W, Jones B, Gonsalez S, Andar U, Hayward R: Subdural intracranial pressure monitoring in craniosynostosis: its role in surgical management. Childs Nerv Syst 11:269-275, 1995

9. Thompson DN, Malcolm GP, Jones BM, Harkness WJ, Hayward RD: Intracranial pressure in single-suture craniosynostosis. Pediatr Neurosurg 22:235-240, 1995

10. Wall SA, Thomas GP, Johnson D, Byren JC, Jayamohan J, Magdum SA, et al: The preoperative incidence of raised intracranial pressure in nonsyndromic sagittal craniosynostosis is underestimated in the literature. J Neurosurg Pediatr 14:674-681, 2014

11. Wayenberg JL, Hasaerts D, Franco P, Valente F, Massager $\mathrm{N}$ : Anterior fontanelle pressure variations during sleep in healthy infants. Sleep 18:223-228, 1995

12. Yokote A, Aihara Y, Eguchi S, Okada Y: Intraoperative preand post-craniofacial reconstruction intracranial pressure (ICP) monitoring in children with craniosynostosis. Childs Nerv Syst 29:1363-1367, 2013

\section{Disclosures}

The authors report no conflict of interest concerning the materials or methods used in this study or the findings specified in this paper.

\section{Author Contributions}

Conception and design: Sood, Rozzelle. Acquisition of data: Sood, Marupudi. Analysis and interpretation of data: Sood, Marupudi, Rozzelle. Drafting the article: all authors. Critically revising the article: all authors. Reviewed submitted version of manuscript: all authors. Approved the final version of the manuscript on behalf of all authors: Sood.

\section{Supplemental Information \\ Previous Presentations}

This paper was presented as part of an oral presentation at the 43rd Annual AANS/CNS Section on pediatric neurological surgery in Amelia Island, Florida, on December 2-5, 2014.

\section{Correspondence}

Sandeep Sood, Pediatric Neurosurgery, Children's Hospital of Michigan, 3901 Beaubien St., 2nd Fl., Detroit, MI 48201. email: ssood@med.wayne.edu. 